\title{
Fachgruppe Computeralgebra: 29-jähriges Bestehen
}

Florian $\mathrm{Heß}$ und Gregor Kemper

Die Fachgruppe Computeralgebra in Deutschland wurde 1987 gegründet. Die Initiative dazu ging u.a. aus von Albrecht Blaser (Heidelberg), Benno Fuchssteiner (Paderborn), Reiner Janßen (Heidelberg), Joachim Neubüser (Aachen) und wurde von Willi Törnig (Darmstadt) und Wolfgang Walter (Karlsruhe) unterstützt. Es galt, der stürmischen Entwicklung dieses Gebietes im Überlappungsbereich von Informatik, Mathematik und Anwendungsgebieten in den Natur-, Ingenieur- und Wirtschaftswissenschaften eine gemeinsame Plattform zu geben. Im späteren Verlauf wurde die Fachgruppe stark geprägt durch Wolfram Koepf, der von 2002 bis 20I I die Sprecherschaft innehatte und die Fachgruppe mit großem Engagement und ruhiger Hand führte. Die Fachgruppe wird getragen von der GI (Gesellschaft für Informatik) in Kooperation mit der DMV und der GAMM (Gesellschaft für angewandte Mathematik und Mechanik).

Die Computeralgebra beschäftigt sich mit Methoden zum Lösen mathematisch formulierter Probleme durch Algorithmen zum symbolischen und algebraischen Rechnen sowie deren Umsetzung in Soft- und Hardware. Computeralgebra beruht auf der exakten, endlichen Darstellung mathematischer Objekte und Strukturen und ermöglicht deren symbolische und formelmäßige Behandlung durch einen Computer. Das Rechnen mit beliebig langen Zahlen, mit Symbolen und Unbestimmten, das Faktorisieren von Zahlen und Polynomen, das symbolische Differenzieren, das Auffinden von Stammfunktionen, das exakte Lösen polynomieller Gleichungssysteme oder von Differentialgleichungen sind einige konkrete Beispiele für Problemstellungen in der Computeralgebra. Die Computeralgebra stellt rechnerische Werkzeuge für die Forschung in Gruppentheorie, Zahlentheorie, kommutativer Algebra, algebraischer Geometrie und Kombinatorik zur Verfügung. Dabei gehen Algorithmenentwicklung, Implementierung sowie theoretische und experimentelle Forschung Hand in Hand. Entsprechend vielfältig sind die Anwendungsbereiche, zu denen neben Naturwissenschaften und Technik auch Kryptologie, Optimierung und Bildverarbeitung gehören. Diese verschiedenartigen Aspekte haben eines gemeinsam: Im Gegensatz zur numerischen Mathematik mit ihrer Gleitkomma-Arithmetik und Rundungsfehlerproblematik stehen hier exakte algebraische Rechnungen sowie die symbolische Manipulation von Formeln im Mittelpunkt.

Die Fachgruppe sieht es als ihre Aufgabe an, Forschung, Lehre und Entwicklung, Anwendungen, Informationsaustausch und Zusammenarbeit auf dem Gebiet der Computeralgebra zu fördern. Zur Umsetzung dieser Ziele übernimmt die Fachgruppe eine Vielzahl von Aufgaben:
- Sie betreibt im Internet eine Homepage unter der URL www.fachgruppe-computeralgebra.de mit interessanten und aktuellen Informationen rund um die Computeralgebra und die Aktivitäten der weltweiten Gemeinde der Computeralgebraiker.

- Sie gibt den Computeralgebra-Rundbrief heraus, der zweimal im Jahr erscheint und an die Mitglieder versandt wird. In diesem finden sich Artikel in verschiedenen Rubriken wie „Themen und Anwendungen der Computeralgebra“, „Neues über Computeralgebra-Systeme“ und „Computeralgebra in der Schule“ sowie Informationen, beispielsweise Konferenzhinweise und -berichte. Der Rundbrief steht mit Ausnahme der jeweils aktuellen Ausgabe auch auf der Homepage zur Verfügung. Im Jahr der Mathematik 2008 erschien ein umfangreiches Sonderheft für die Zielgruppe Schüler und Lehrer, das an allen Gymnasien in Deutschland verteilt wurde.

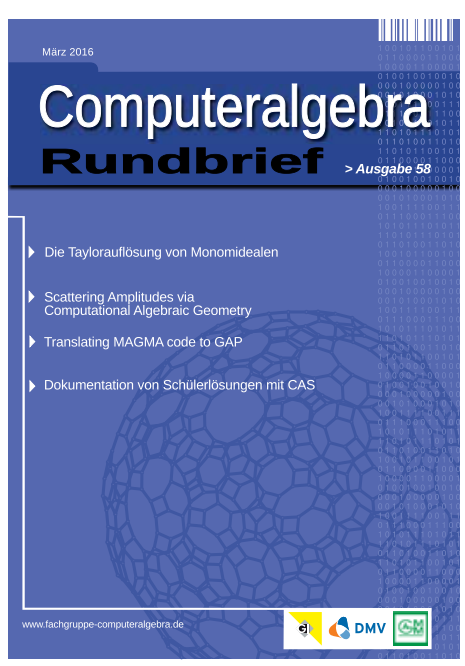

- Die Fachgruppe organisiert und unterstützt Workshops, Seminare, Tagungen und andere Aktivitäten auf dem Gebiet der Computeralgebra. So wurde in den Jahren 2003, 2005, 2007, 2009, 2012 und 2014 jeweils eine dreitägige Computeralgebra-Tagung veranstaltet, bei der es neben eingeladenen Hauptvorträgen etablierter Wissenschaftler zahlreiche Doktorandenvorträge sowie mehrere Software-Präsentationen gab. Für den besten Vortrag eines Nachwuchswissenschaftlers wird regelmäBig ein Preis in Höhe von 500 Euro vergeben. Die nächste Computeralgebra-Tagung wird im Jahr 2017 stattfinden.

Auch internationale Konferenzen finden unter Beteiligung der Fachgruppe statt. So konnte dreimal das renommierte "International Symposium on Symbolic and Algebraic Computation (ISSAC)“" nach Deutschland geholt 


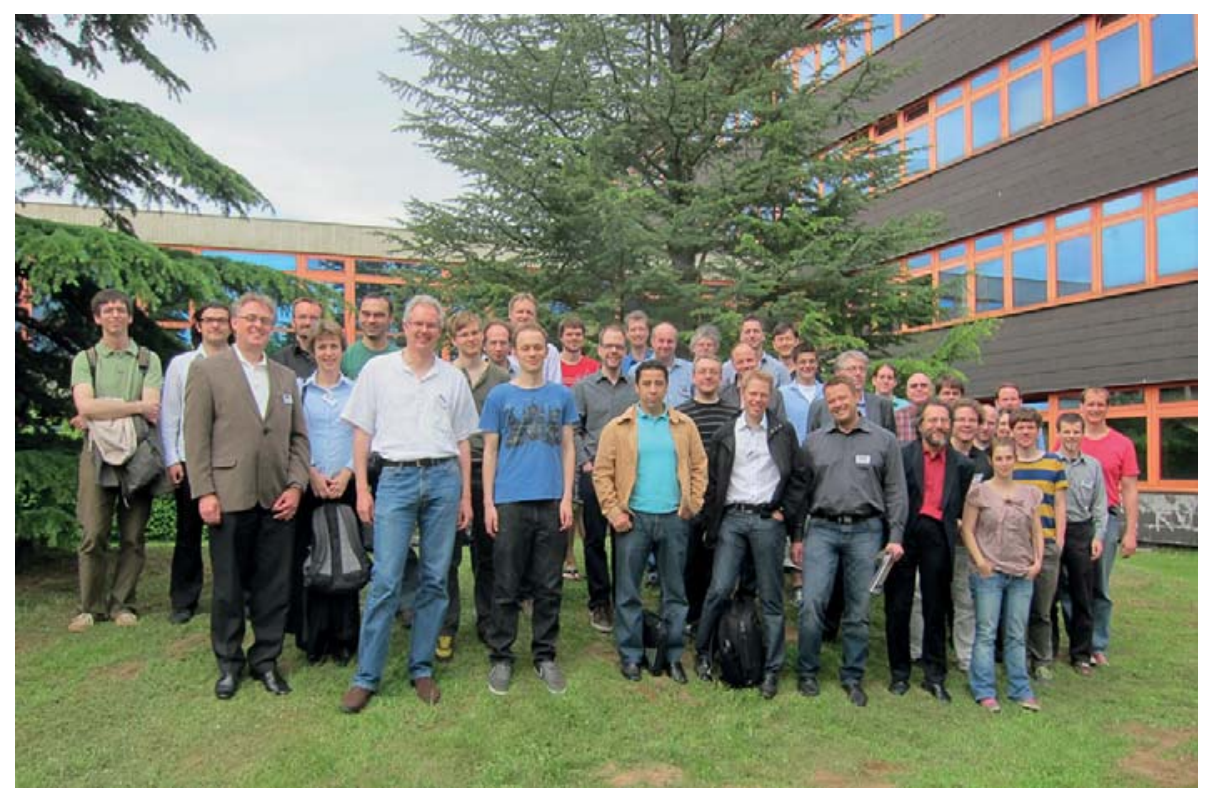

Computeralgebra-Tagung 2012 (Foto: Privat)

werden, und zwar 199I nach Bonn, 1998 nach Rostock und 2010 nach München. Im Jahr 2017 wird das ISSAC in Kaiserslautern stattfinden. Auch beim ISSAC verleiht die Fachgruppe Preise für das beste Poster und die beste Software-Demonstration. Im Jahr $201 \mathrm{I}$ organisierte die Fachgruppe eine Tagung, die sich insbesondere an Anwender von Computeralgebra in der Industrie wandte. Die Fachgruppe beteiligt sich auch regelmäßig bei der Gestaltung der Jahrestagungen ihrer Trägerorganisationen, etwa durch Sektionen oder Minisymposien.

- Die Nachwuchsförderung ist ein wichtiges Anliegen der Fachgruppe. Neben der Verleihung der oben genannten Preise beteiligt sich die Fachgruppe aktiv an der Diskussion um die Stellung der Computeralgebra in der Ausbildung in Schule und Hochschule, zum Beispiel im „Arbeitskreis Mathematikunterricht und Informatik“ der GDM (Gesellschaft für Didaktik der Mathematik). Gerade in der Schulausbildung wird der Einsatz von Computeralgebra heftig diskutiert.

Die Koordination dieser Aktivitäten und die Herausgabe des Rundbriefs liegt in den Händen der Fachgruppenleitung, die hierzu jedes Jahr im Februar und September an wechselnden Orten zusammenkommt. Dieses Gremium hat mindestens zwölf Mitglieder, die sich neben der kollegialen Leitung der Fachgruppe jeweils um bestimmte Aufgaben kümmern, die zum Teil den Rubriken des Rundbriefs entsprechen. Drei Mitglieder der Fachgruppenleitung werden von der GI, DMV und GAMM als Vertreter benannt; Vertreter der DMV ist Wolfram Koepf (Kassel), der auch dem Präsidium der DMV angehört. Bis zu drei weitere können als Fachexperten berufen werden, und die restlichen neun werden von sämtlichen Mitgliedern der Fachgruppe gewählt. Aktuell sind zehn der I4 Mitglieder der Fachgruppenleitung auch Mitglieder der
DMV, was die inhaltliche Verankerung der Fachgruppe in der DMV unterstreicht. Die Amtszeit der Fachgruppenleitung beträgt drei Jahre. Die gewählten Sprecher der Fachgruppe sind aktuell Gregor Kemper (München) und Florian $\mathrm{He}$ (Oldenburg).

Wir laden alle an Computeralgebra Interessierten zur Teilhabe und Mitwirkung an der Fachgruppe ein. Die Mitgliedschaft in der Fachgruppe steht allen Interessierten offen. Die Fachgruppe strebt dabei an, dass jedes ihrer Mitglieder auch Mitglied in der GI, DMV oder GAMM ist. Der Jahresbeitrag beträgt 7,50 Euro für Mitglieder einer der Trägergesellschaften, sonst 9 Euro. Zurzeit hat die Fachgruppe knapp 400 Mitglieder.

Weitere Informationen:

www.fachgruppe-computeralgebra.de

Prof. Dr. Florian Heß, Carl von Ossietzky Universität Oldenburg, Institut für Mathematik, 26I I I Oldenburg

florian.hess@uni-oldenburg.de

Prof. Dr. Gregor Kemper, Technische Universität München, Zentrum Mathematik - MII, Boltzmannstraße 3, 85748 Garching bei München. kemper@ma.tum.de

Florian $\mathrm{He} ß$ studierte Mathematik in Berlin, wo er 1999 promovierte. Von 2003 bis 2009 und 2009 bis 2010 hatte er Professuren in Berlin und Magdeburg inne. Seit 2010 ist er Professor in Oldenburg. Sein Forschungsinteresse gilt der algorithmischen algebraischen Zahlentheorie und Geometrie sowie ihren Anwendungsbereichen

Gregor Kemper studierte Mathematik in Karlsruhe und Heidelberg, wo er 1994 promovierte. Seit 2002 ist er Professor für algorithmische Algebra an der Technischen Universität München. Sein Forschungsinteresse gilt der Invariantentheorie und der kommutativen Algebra.
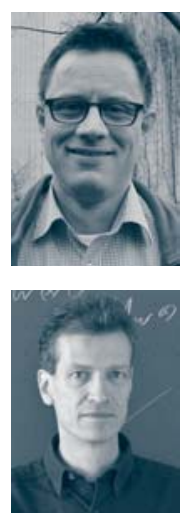